\title{
Polarization-Entangled Photon Pairs From Periodically-Poled Crystalline Waveguides Over a Range of Frequencies
}

\author{
Dylan A. Heberle ${ }^{1,2}$ and Zachary H. Levine ${ }^{2}$ \\ ${ }^{1}$ Rochester Institute of Technology, \\ 1 Lomb Memorial Drive, \\ Rochester, NY 14623-5603 \\ ${ }^{2}$ National Institute of Standards and Technology, \\ Gaithersburg, MD 20899-8441 \\ zachary.levine@nist.gov
}

We propose a method to extend the frequency range of polarization entanglement in periodically poled rubidium-doped potassium titanyl phosphate (Rb:KTP) waveguides. Our calculations predict that output wavelengths from $1130 \mathrm{~nm}$ to $1257 \mathrm{~nm}$ may be achieved using Rb:KTP by the appropriate selection of a direction of propagation for the waveguide. The fidelity using a poling period of $1 \mathrm{~mm}$ is approximately 0.98 .

Key words: entangled photons; frequency and angle tuning; periodically-poled waveguide; Rb:KTP (rubium doped potassium titanyl phosphate).

Accepted: July 24, 2013

Published: August 15, 2013

http://dx.doi.org/10.6028/jres.118.018

\section{Introduction}

Photon entanglement is a requirement for various applications in nonlinear optics and quantum mechanics [1,2]. Polarization entanglement has been implemented in areas such as quantum teleportation [3], quantum informatics [4], and quantum cryptography [5]. Recently, entangled states have been used in the design of quantum circuitry using domain-engineering [6,7]. Another recent study demonstrated that high spatial entanglement was possible using nonlinear waveguide arrays [8].

Other studies have focused on the development of entangled photon pairs using periodically poled crystals and waveguides. One of the most commonly poled materials is potassium titanyl phosphate (KTP). Kim et al. [9] placed periodically poled KTP (PPKTP) inside a Sagnac interferometer to produce entangled photon pairs. Periodically poled lithium niobate (PPLN) has been used to achieve polarization-entangled photon pairs emitted at the telecom wavelength [10] and to demonstrate the steering of entangled photons [6]. Recently, the generation of entangled photons directly from an optical fiber has been demonstrated, albeit with low source intensity [11]. 
Our group proposed a method [12] which, like Ref. [11], allows for the direct generation of polarization-entangled photon pairs. However, the proposed method was restricted to operation at very few frequencies. To realize a range of quasi-phase-matched operational frequencies for type-II spontaneous parametric downconversion (SPDC) in periodically poled waveguides, we propose orienting the waveguide at an angle relative to the crystalline structure during fabrication. As different orientations of the waveguide are selected, the effective indices of refraction are changed, allowing a range of frequencies to be quasiphase matched. We take advantage of the biaxial nature of KTP to permit this reorientation. Although the fabrication of PPKTP waveguides at an arbitrary orientation does not appear to have been achieved, lateral patterning of periodically poled lithium niobate[13] and lithium tantalate [6] waveguides have been realized experimentally.

\section{Theoretical Development}

Polarization entanglement can be achieved using type-II SPDC in periodically poled waveguides. In this process, a high-frequency photon, called the pump photon, is downconverted into two lower frequency photons, known as the signal and idler. The pump photon and either the signal or the idler photon are polarized in the same direction which is orthogonal to the polarization of the third photon. In the previous study [12], periodic poling was used to achieve quasi-phase matching for two different type-II SPDC processes simultaneously. The poling period $\Lambda$ was used to offset the wave vector mismatch $\Delta k$ given by

$$
\Delta k_{m}\left(\omega_{s}, \omega_{i}\right)=k_{V}\left(\omega_{s}\right)+k_{H}\left(\omega_{i}\right)-k_{H}\left(\omega_{p}\right)+\frac{2 \pi m}{\Lambda}
$$

where $\omega_{p}, \omega_{s}$, and $\omega_{i}$ are the angular frequencies of the pump, signal, and idler photons respectively and $V$ and $H$ stand for the vertical and horizontal polarizations. A polarization-entangled state was achieved by quasi-phase matching the $m= \pm 1$ modes, as shown by the following equations

$$
\begin{aligned}
& \Delta k_{1}\left(\omega_{s}, \omega_{i}\right)=k_{V}\left(\omega_{s}\right)+k_{H}\left(\omega_{i}\right)-k_{H}\left(\omega_{p}\right)+\frac{2 \pi}{\Lambda}=0 \\
& \Delta k_{-1}\left(\omega_{i}, \omega_{s}\right)=k_{V}\left(\omega_{i}\right)+k_{H}\left(\omega_{s}\right)-k_{H}\left(\omega_{p}\right)-\frac{2 \pi}{\Lambda}=0 .
\end{aligned}
$$

Our scheme uses the same entanglement mechanism but proposes a selection of the waveguide orientation during fabrication to achieve a range of operational frequencies.

For bulk KTP and Rb:KTP waveguides, the crystal $Z$ axis needs to be aligned normal to the surface so that the standard diffusion process may occur. However, we consider propagation directions of the waveguide at an angle $\phi$ in the crystal $X Y$ plane, with $\phi=0$ corresponding to propagation along the $X$ axis.

The indices of refraction for KTP and Rb:KTP are calculated from the Sellmeier equations of König and Wong [14], who introduce a new Sellmeier equation for $n_{Y}^{(K T P)}$ and suggest the Sellmeier equation of Fradkin et al. [15] for $n_{z}^{(K T P)}$. To find the difference between the indices of KTP and RTP (rubidium titanyl phosphate) for the waveguides, we use the Sellmeier equations from Cheng et al. [16] who report the values for both crystals using one set of instruments. In this study, we introduce a new Sellmeier equation for $n_{X}^{(K T P)}$ given by

$$
n_{X}^{(K T P)}=n_{Y}^{(\text {KTP,König })}-n_{Y}^{(K T P, \text { Cheng })}+n_{X}^{(K T P, \text { Cheng })} .
$$


This equation is formulated to approximate the value of $n_{X}^{(K T P)}$ so that it agrees with the values of $n_{Y}^{(K T P)}$ and $n_{Z}^{(K T P)}$ from König and Wong which were developed for PPKTP.

The effective indices of refraction are calculated using the Sellmeier equations given above combined with a tensor transformation, which rotates the inverse dielectric tensor clockwise by an angle $\phi$ as previously defined. Using the commercial software package Comsol Multiphysics and its RF (Radio Frequency) Module [17], we calculate the effective indices of refraction in certain waveguides over a range of orientations. We use the simulation region and diffusion profile from Levine et al. [12]. This region consists of a doped-rectangular area of width $w$ between two quarter circles of KTP with $20 \mu \mathrm{m}$ radii and a $(40 \mu \mathrm{m}+w) \times 4 \mu \mathrm{m}$ rectangular region of air on top. The index profile follows the function erfc $\left(-Z / Z_{0}\right)$ [18] as it decreases from the bulk RTP value at the crystal surface, $Z=0$, to the bulk KTP value within the waveguide, $Z \leq 0$.

The effective indices of refraction are shown in Fig. 1. Although the crystal is nearly uniaxial, the splitting between the $X$ and $Y$ polarizations leads to significant tuning of the operational wavelengths.

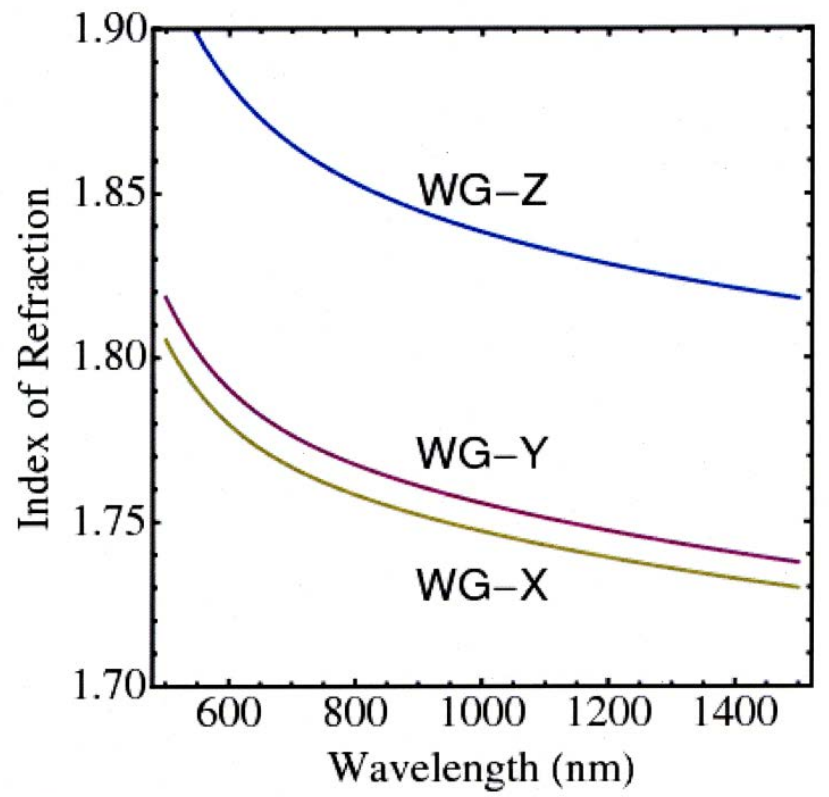

Fig. 1. (Color online) The effective indices of refraction for the $3.5 \mu \mathrm{m} \times 4.5 \mu \mathrm{m}$ Rb:KTP waveguide described in the text are given for the three crystal axes.

\section{Simulation Results}

The effective indices of refraction are used to find the phase-matched wavelengths resulting in polarization-entangled type-II SPDC in periodically poled Rb:KTP waveguides. We study a $3.5 \mu \mathrm{m} \times$ $4.5 \mu \mathrm{m} \mathrm{Rb}: \mathrm{KTP}$ waveguide, where the dimensions are chosen so that the signal and idler will have a single mode in the waveguide. We find that the degenerate output wavelength decreases from $1257 \mathrm{~nm}$ at $\phi=0^{\circ}$ to $1130 \mathrm{~nm}$ at $\phi=90^{\circ}$, as shown in the right sides of the graphs in Fig. 2. Our operating point involves satisfying both parts of (2), so Fig. 2 differs from a typical tuning curve. The phase-matched frequencies decrease as $\phi$ increases because the crystal $Y$ index of refraction is greater than the crystal $X$ index of refraction for KTP. The quasi-phase-matched wavelengths for type-II SPDC in bulk KTP and various Rb:KTP waveguides using a poling period of $\Lambda=1 \mathrm{~mm}$ are presented in Table 1 . Because this study uses collinear propagation, the bulk case is only a theoretical exercise. 

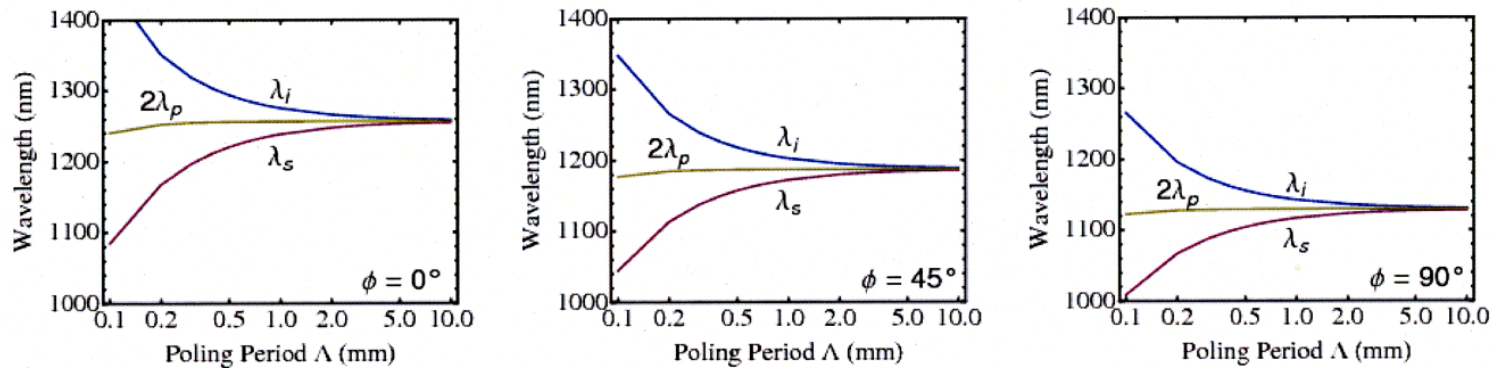

Fig. 2. (Color online) The signal, idler, and pump wavelengths $\left(\lambda_{s}, \lambda_{i}\right.$, and $\lambda_{p}$, respectively) as a function of poling period for $3.5 \mu \mathrm{m} \times 4.5 \mu \mathrm{m} \mathrm{Rb}: \mathrm{KTP}$ waveguide for various orientations. As the orientation angle $\phi$ is increased, the quasi-phase-matched frequency for each poling period decreases. The $\phi=0^{\circ}$ case was presented previously [12].

Table 1. Phase-matched signal, idler, and pump wavelengths $\left(\lambda_{s}, \lambda_{i}\right.$, and $\lambda_{p}$, respectively) using a poling period of $1 \mathrm{~mm}$ in Rb:KTP waveguides and bulk KTP

\begin{tabular}{cccccc}
\hline \multirow{2}{*}{$w(\mu \mathrm{m})$} & $Z_{0}(\mu \mathrm{m})$ & $\phi$ & $\lambda_{s}(\mathrm{~nm})$ & $\lambda_{i}(\mathrm{~nm})$ & $\lambda_{p}(\mathrm{~nm})$ \\
\hline \multirow{3}{*}{3.5} & \multirow{3}{*}{4.5} & $0^{\circ}$ & 1239 & 1275 & 628 \\
& & $45^{\circ}$ & 1172 & 1203 & 594 \\
& $90^{\circ}$ & 1117 & 1142 & 565 \\
\hline \multirow{3}{*}{5.0} & \multirow{3}{*}{6.0} & $0^{\circ}$ & 1212 & 1247 & 615 \\
& & $45^{\circ}$ & 1156 & 1185 & 585 \\
& $90^{\circ}$ & 1110 & 1135 & 561 \\
\hline \multirow{3}{*}{ bulk KTP } & & $0^{\circ}$ & 1069 & 1093 & 540 \\
& & $45^{\circ}$ & 1025 & 1047 & 518 \\
& $90^{\circ}$ & 985 & 1003 & 497 \\
\hline
\end{tabular}

To find the effect of angular dependence on efficiency, we calculated the effective nonlinear coefficients for type-II phase matching in KTP given by Ref. [19] where $\left|d_{15}\right|=1.2 \mathrm{pm} / \mathrm{V}$ and $\left|d_{24}\right|=$ $2.2 \mathrm{pm} / \mathrm{V}$, where a small dispersion correction has been included. An additional factor of $2 / \pi$ is required to account for quasi-phase matching vs. standard phase matching [20]. The effective nonlinearity in the $X Y$ plane of KTP is given by

$$
d_{\text {eoe }}=d_{\text {oee }}=d_{15} \sin ^{2} \phi+d_{24} \cos ^{2} \phi .
$$

The efficiency may be calculated using Eq. (6) of Ref. [21] enhanced by a factor of 2 to account for the two processes. We choose a crystal length of $36 \mathrm{~mm}$ to match Ref. [22] and calculate at quasi-phase matching a maximum rate of $4 \cdot 10^{6} \mathrm{~s}^{-1} \mathrm{GHz}^{-1} \mathrm{~mW}^{-1}$ to $1 \cdot 10^{6} \mathrm{~s}^{-1} \mathrm{GHz}^{-1} \mathrm{~mW}^{-1}$ as $\phi$ varies from $0^{\circ}$ to $90^{\circ}$, with a full-width at half-maximum (FWHM) bandwidth which decreases roughly linearly from $1.3 \mathrm{~nm}$ to $0.7 \mathrm{~nm}$ in this angular range. This compares to a value of $3 \cdot 10^{5} \mathrm{~s}^{-1} \mathrm{GHz}^{-1} \mathrm{~mW}^{-1}$ in a recent experiment using a PPLN waveguide followed by discrete optical components [22], suggesting the present scheme is potentially a competitive entangled photon source.

Fidelity is a measure of the utility of the states for use in two-photon interference experiments and other quantum information applications [1,2]. For example, a fidelity value of $F>0.78$ is required to satisfy the Clauser-Horne-Shimony-Holt inequality $[23,24]$ which was proposed for a loophole-free version of Bell's inequality. Such fidelity has been demonstrated experimentally including $F=0.82$ by Santori et al. [25], $F=0.99$ by Peters et al. [26], $F>0.92$ by Politi et al. [27], and $F=0.97$ by Ling et al. [28]. 
Here, we find that across the orientations, the waveguides maintain the high fidelity calculated by Levine et al. [12], as shown in Fig. 3. As in the earlier work, the predictions are subject to certain numerical approximations which limit the maximum predicted fidelity to $0.99 \pm 0.01$ at $1 \mathrm{~mm}$, with increasing uncertainty for the longer poling periods.

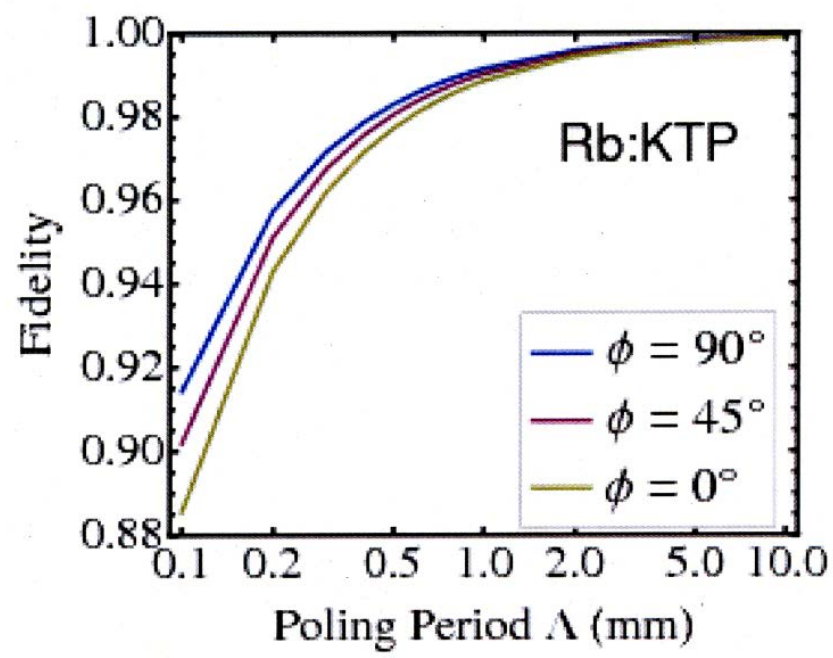

Fig. 3. (Color online) Fidelity as a function of poling period for the $3.5 \mu \mathrm{m} \times 4.5 \mu \mathrm{m} \mathrm{Rb}: \mathrm{KTP}$ waveguide. The $\phi=0^{\circ}$ line (bottom curve) for the Rb:KTP waveguide is the result found by Levine et al. [12].

\section{Concluding Remarks}

We propose a scheme which allows the generation of entangled photons using type-II SPDC in periodically poled waveguides over a range of frequencies. By orienting the waveguide relative to the crystal axes, we predict a degenerate output wavelength range of $1130 \mathrm{~nm}$ to $1257 \mathrm{~nm}$ in an unpoled $3.5 \mu \mathrm{m} \times 4.5 \mu \mathrm{m} \mathrm{Rb}: \mathrm{KTP}$ waveguide. Other high-efficiency operation points may be achieved by using other biaxial materials such as potassium titanyl arsenate (KTA).

The proposed waveguides maintain a moderate efficiency and high fidelity regardless of the orientation of the waveguide relative to the crystal axes. By introducing a new degree of freedom, polarizationentangled type-II SPDC may operate over a broader range of wavelengths.

\section{Acknowledgments}

We thank Jingyun Fan, Paulina Kuo, and Alan Migdall for useful discussions.

\section{References}

[1] R. Horodecki, P. Horodecki, M. Horodecki, and K. Horodecki, Quantum entanglement, Rev. Mod. Phys. 81, 865-942 (2009).

[2] J.-W. Pan, Z.-B. Chen, C.-Y. Lu, H. Weinfurter, A. Zellinger, and M. Żukowski, Multiphoton entanglement and interferometry, Rev. Mod. Phys. 84, 777-838 (2012).

[3] D. Bouwmeester, J.-W. Pan, K. Mattle, M. Eibl, H. Weinfurter, and A. Zeilinger, Experimental quantum teleportation, Nature 390, 575-579 (1997). http://dx.doi.org/10.1038/37539

[4] C. H. Bennett and D. P. DiVincenzo, Quantum information and computation, Nature 404, 247-255 (2000).

[5] A. K. Ekert, J. G. Rarity, P. R. Tapster, and G. M. Palma, Practical quantum cryptography based on two-photon interferometry, Phys. Rev. Lett. 69, 1293-1295 (1992). http://dx.doi.org/10.1103/PhysRevLett.69.1293

[6] H. Y. Leng, X. Q. Yu, Y. X. Gong, P. Xu, Z. D. Xie, H. Jin, C. Zhang, and S. N. Zhu, On-chip steering of entangled photons in nonlinear photonic crystals, Nat. Commun. 2, 429 (2011). 
[7] X. Q. Yu, P. Xu, Z. D. Xie, J. F. Wang, H. Y. Leng, J. S. Zhao, S. N. Zhu, and N. B. Ming, Transforming spatial entanglement using a domain-engineering technique, Phys. Rev. Lett. 101, 233601 (2008).

[8] A. S. Solntsev, A. A. Sukhorukov, D. N. Neshev, and Y. S. Kivshar, Spontaneous parametric down-conversion and quantum walks in arrays of quadratic nonlinear waveguides, Phys. Rev. Lett. 108, 023601 (2012).

[9] T. Kim, M. Fiorentino, and F. N. C. Wong, Phase-stable source of polarization-entangled photons using a polarization Sagnac interferometer, Phys. Rev. A 73, 012316 (2006).

[10] A. Martin, A. Issautier, H. Herrmann, W. Sohler, D. B. Ostrowsky, O. Alibart, and S. Tanzilli, A polarization entangled photonpair source based on a type-II PPLN waveguide emitting at a telecom wavelength, New J. Phys. 12, 103005 (2010).

[11] E. Y. Zhu, Z. Tang, L. Qian, L. G. Helt, M. Liscidini, J. E. Sipe, C. Corbari, A. Canagasabey, M. Ibsen, and P. G. Kazansky, Direct generation of polarization-entangled photon pairs in a poled fiber, Phys. Rev. Lett. 108, 213902 (2012).

[12] Z. H. Levine, J. Fan, J. Chen, and A. L. Migdall, Polarization-entangled photon pairs from a periodically poled crystalline waveguide, Optics Express 19, 6724-6740 (2011).

[13] G. Imeshev, M. Proctor, and M. M. Fejer, Lateral patterning of nonlinear frequency conversion with transversely varying quasiphase-matching gratings, Opt. Lett. 23, 673-675 (1998).

[14] F. König and F. N. C. Wong, Extended phase matching of second-harmonic generation in periodically poled $\mathrm{KTiOPO}_{4}$ with zero group velocity mismatch, Appl. Phys. Lett. 84, 1644-1646 (2004).

[15] K. Fradkin, A. Arie, A. Skliar, and G. Rosenman, Tunable midinfrared source by difference frequency generation in bulk periodically poled $\mathrm{KTiOPO}_{4}$, Appl. Phys. Lett. 74, 914-916 (1999).

[16] L. K. Cheng, L. T. Cheng, J. Galperin, P. A. M. Hotsenpiller, and J. D. Bierlein, Crystal-growth and characterization of $\mathrm{KTiOPO}_{4}$ isomorphs from the self-fluxes, J. Cryst. Growth 137, 107-115 (1994). http://dx.doi.org/10.1016/0022-0248(94)91256-4

[17] http://www.comsol.com. Mention of commercial products is for information only; it does not imply recommendation or endorsement by NIST.

[18] J. D. Bierlein, A. Ferretti, L. H. Brixner, and W. Y. Hsu, Fabrication and characterization of optical waveguides in $\mathrm{KTiOPO}_{4}$ Appl. Phys. Lett. 50, 1216-1218 (1987).

[19] H. Vanherzeele and J. D. Bierlin, Magnitude of the nonlinear-optical coefficients of $\mathrm{KTiOPO}_{4}$, Opt. Lett. 17, 982-984 (1992).

[20] M. M. Fejer, G. A. Magel, D. H. Jundt, and R. L. Byer, Quasi-phase-matched second harmonic generation: tuning and tolerances, IEEE J. Quant. Electron. 28, 2631-2654 (1992).

[21] S. M. Spillane, M. Fiorentino, and R. G. Beausoleil, Spontaneous parametric down conversion in a nanophotonic waveguide, Optics Express 15, 8770-8780 (2007).

[22] A. Martin, V. Cristofori, P. Aboussouan, H. Herrmann, W. Sohler, D. B. Ostrowsky, O. Alibart, and S. Tanzilli, Integrated optical source of polarization entangled photons at $1310 \mathrm{~nm}$, Optics Express 17, 1033-1041 (2009).

[23] J. F. Clauser, M. A. Horne, A. Shimony, and R. A. Holt, Proposed experiment to test local hidden-variable theories, Phys. Rev. Lett. 23, 880-884 (1969). http://dx.doi.org/10.1103/PhysRevLett.23.880

[24] C. H. Bennett, G. Brassard, S. Popescu, B. Schumacher, J. A. Smolin, and W. K. Wooters, Purification of noisy entanglement and faithful teleportation via noisy channels, Phys. Rev. Lett. 76, 722-725 (1996). http://dx.doi.org/10.1103/PhysRevLett.76.722

[25] C. Santori, D. Fattal, J. Vučković, G. S. Soloman, and Y. Yamamoto, Indistinguishable photons from a single-photon device, Nature 419, 594-597 (2002).

[26] N. A. Peters, J. B. Altpeter, D. Branning, E. R. Jeffrey, T. C. Wei, and P. G. Kwiat, Maximally entangled mixed states: creation and concentration, Phys. Rev. Lett. 92, 133601 (2004). http://dx.doi.org/10.1016/j.contraception.2003.10.007

[27] A. Politi, M. J. Cryan, J. G. Rarity, S. Y. Yu, and J. L. O’Brien, Silica-on-silicon waveguide quantum circuits, Science 320 646-649 (2008).

[28] A. Ling, J. Chen, J. Y. Fan, and A. Migdall, Mode expansion and Bragg filtering for a high-fidelity fiber-based photon-pair source, Optics Express 42, 21302-21312 (2009).

About the authors: Dylan A. Heberle holds a B.S. degree Physics from the Rochester Institute of Technology and will be a graduate student in Engineering Physics at Cornell University. He was a NIST Summer Undergraduate Research Fellow. Zachary H. Levine is a Physicist in the Sensor Science Division at NIST. The National Institute of Standards and Technology is an agency of the U.S. Department of Commerce. 